\title{
SERUM LIPID PROFILE- HOW IT ALTERS IN NORMOTENSIVE AND HYPERTENSIVE PREGNANT WOMEN
}

Dhrubajyati Saha1, Pampa Roy², Rajib Pal ${ }^{3}$, Tapan Kumar Ganguli4, Santu Maity ${ }^{5}$, Bibek Mohan Rakshit ${ }^{6}$, Prabir Sengupta7 ${ }^{7}$ Sanjana Halder ${ }^{8}$

HOW TO CITE THIS ARTICLE:

Dhrubajyati Saha, Pampa Roy, Rajib Pal, Tapan Kumar Ganguli, Santu Maity, Bibek Mohan Rakshit, Prabir Sengupta, Sanjana Halder. "Serum lipid profile- how it alters in normotensive and hypertensive pregnant women". Journal of Evolution of Medical and Dental Sciences 2013; Vol2, Issue 31, August 5; Page: 5895-5902.

ABSTRACT: OBJECTIVES: The present study was designed to evaluate the association of lipid profile in pre- eclampsia and if there is any change in lipid profiles in pre-eclampsic mother as compared to no pregnant woman and normotensive pregnant mother. METHODS: This case-control study was carried out in the department of Obstetrics and gynaecology of Burdwan medical college, Burdwan from $1^{\text {st }}$ June 2012 to 31 ${ }^{\text {st }}$ May 2013.Total 180 patient were evaluated of which 60 were non-pregnant normotensive,60 were pregnant normotensive and 60 were pre-eclamptic mother. Age group was in between 19-35 years. In case of pregnancy gestational age was in between 32 to 36 weeks. Patients with pre-existing hypertension were excluded. Serum lipid profile (total cholesterol, triglycerides, HDL-cholesterol VLDL and LDL cholesterol) of one hundred and eighty women with Pre-eclampsia $(n=60)$, normotensive non pregnant women $(n=60)$ and normotensive pregnant woman $(n=60)$ were monitored .Comparison is drawn and analysed by using chi-square test (SPSS 18 software). RESULTS: In preeclampsia there is significant decrease of High density lipoprotein(HDL) and significantly increase of Low density lipoprotein(LDL),Very low density lipoprotein (VLDL) and Triglycerides concentration seen compared to non pregnant normotensive and pregnant normotensive subjects. CONCLUSION: Lipid metabolism plays a key role in the pathophysiology of Pre-eclampsia and Eclampsia. Increased triglycerides, total cholesterol, LDL, VLDL levels along with decreased HDL-cholesterol levels and high blood pressure are associated with development of preeclampsia and eclampsia. This association may be significant in understanding the pathologic processes of preeclampsia and may help in developing strategies for prevention or early diagnosis of this disorder.

KEYWORDS: Lipid Profile, Preeclampsia

INTRODUCTION: Hypertension during pregnancy is a major health problem. It is one of the leading causes of perinatal morbidity and mortality. (1) Preeclampsia (PE) is a theoretical disease with a pathogenesis that is not clearly understood yet. Lately vascular system pathology and vasoconstriction have been blamed as causes for preeclampsia with growing acceptance. Lipid values in normal pregnancies change with gestational age. In a normotensive pregnancy, serum triglyceride, total and HDL-cholesterol increase during pregnancy, but lipoprotein A levels decrease. These changes are reported to be secondary to hormonal changes during pregnancy. (1-3)

The most important feature in toxaemia of pregnancy is hypertension which is supposed to be due to vasospastic phenomenon in kidney, uterus, placenta and brain (4). Altered lipid synthesis leading to decrease in $\mathrm{PGI}_{2}$ : TXA2 ratio is also supposed to be an important way of pathogenesis in pregnancy induced hypertension (5). Thus abnormal lipid metabolism seems important in the pathogenesis of pregnancy induced hypertension (PIH) too. 


\section{ORIGINAL ARTICLE}

One important theory of preeclampsia is oxidative stress and abnormal lipid profiles may have role in promotion of oxidative stress and vascular dysfunction .Low density lipoprotein (LDL) and vascular cell adhesion molecule (VCAM) are increased in association with dyslipidemia in pregnancy Obviously the association of serum lipid profile with gestational proteinuric hypertension is highly suggested to reflect some new diagnostic tools.

Pre-eclampsia and eclampsia is associated with substantial risks for the fetus. These include intrauterine growth restriction, death and prematurity with attendant complications where as the mother is at risk of renal failure, pulmonary edema, stroke, and death. Despite considerable research, there are no clinically useful screening tests to identify women in whom it will develop (7). Early pregnancy dyslipidemia is associated with an increased risk of Pre-eclampsia (8). Women with a history of pre-eclampsia have significant differences in lipid parameters and an increased susceptibility to lipoprotein oxidation when compared with women who had normal pregnancy. Disorders of lipoprotein metabolism are reported to be a major cause of hypertension and proteinuria in Pre-eclampsia (6). In view of the above findings it is postulated that alteration of lipid metabolism may play a key role in the development of symptoms of Pre-eclampsia and Eclampsia.

The present study was designed to investigate the alteration in lipid profile (Cholesterol, triglycerides, HDL-cholesterol, LDL -cholesterol and VLDL-cholesterol) in normal pregnancy and pre-eclamptic women. An abnormal lipid profile is known to be strongly associated with atherosclerotic cardiovascular diseases and has a direct effect on endothelial dysfunction.

MATERIAL AND METHOD: The present study was a case control study carried out on pregnant women attended or admitted in Obstetrics and Gynaecology Department of Burdwan Medical College .This study was carried out over a period of 1 year starting from $1^{\text {st }}$ June 2012 to $31^{\text {st }}$ May 2013 after approval from the Ethical Committee of this Institution. Woman $(n=180)$ were placed in three following groups.

Group A ( $n=60)$ - Non-Pregnant normotensive women of peak childbearing age 19-35 years.

Group B $(n=60)$ - Pregnant women without preeclampsia between 32 and 36 weeks of gestation.

Group C $(n=60)$ - Antenatal subjects of gestational age between 32 and 36 weeks with mild/severe preeclampsia .This group constitutes' cases' (Blood pressure $>140 / 90 \mathrm{mmHg}$ with proteinuria, with or without pathological oedema).

Informed consent was taken from all the mothers. Blood samples (about $10 \mathrm{ml}$ of venous blood from antecubital vein using all aseptic precaution) were drawn from all the subjects following a fast of 12 hours and analysed for Serum Triglycerides (TG), Total cholesterol (TC) and HDL cholesterol by enzymatic methods with the help of Glaxo kits on ERBA Chem-5 semi auto analyser Serum LDL cholesterol was calculated by Frederickson-Friedwald's formula according to which LDL cholesterol $=$ Total cholesterol - (HDL cholesterol + VLDL cholesterol). VLDL cholesterol was calculated as $1 / 5$ of Triglycerides. Data were statistically analysed by chi-square test and significance was expressed in term of ' $P$ ' value. Data were analysed by using SPSS Software version 18. 
Those patient who had Diabetic mellitus ,Chronic Hypertension ,Hepatitis ,Medical disorders like renal disease, Haemoglobin $<8$ gm $\%$,BMI>30.Patient treated for PCOS in past were excluded from the study.

RESULTS: In this study, woman of reproductive age group that is between 19 and 35 years were included . The age wise distribution has been shown in table 1. Most of the subjects were between 19 and 26 years as this is the peak child bearing age at our set up.

Table 1: Age wise distribution of subjects in various groups

\begin{tabular}{|c|c|c|c|c|c|c|}
\hline \multirow{2}{*}{ Age(yrs) } & \multicolumn{2}{|c|}{ Group A } & \multicolumn{2}{|c|}{ Group B } & \multicolumn{2}{|r|}{ Group C } \\
\hline & No & Percentage & No & Percentage & No & Percentage \\
\hline $19-22$ & 25 & $41.7 \%$ & 28 & $46.67 \%$ & 31 & $51.7 \%$ \\
\hline $23-26$ & 15 & $25 \%$ & 19 & $31.7 \%$ & 18 & $30 \%$ \\
\hline $27-30$ & 9 & $15 \%$ & 11 & $18.3 \%$ & 7 & $11.7 \%$ \\
\hline $31-34$ & 10 & $16.7 \%$ & 1 & $1.6 \%$ & 0 & 0 \\
\hline 35 & 1 & $1.7 \%$ & 1 & $1.6 \%$ & 4 & $6.7 \%$ \\
\hline
\end{tabular}

RELIGION: Maximum subjects in this study were Hindu because of Hindu dominated area. The incidence of religion amongst various groups was not significant ( $\mathrm{P}>0.05$ by Chi-Square Test) $P$ value for A \& B is $0.418, A \& C$ is 0.232 and for B \& C is 0.699 .

Table 2: Distribution of religion in the groups

\begin{tabular}{|c|c|c|c|c|c|c|}
\hline \multirow{2}{*}{ Religion } & \multicolumn{2}{|c|}{ Group A } & \multicolumn{2}{c|}{ Group B } & \multicolumn{2}{c|}{ Group C } \\
& No & Percentage (\%) & No & Percentage (\%) & No & Percentage (\%) \\
\hline Hindu & 45 & 75 & 41 & 68.33 & 39 & 65 \\
\hline Muslim & 15 & 25 & 19 & 31.66 & 21 & 35 \\
\hline
\end{tabular}

BLOOD PRESSURE: Mean blood pressure of different group is shown bellow .The p value of A\&B is 0.295 for SBP and 0.108 for DBP that is not significant. But p value for B \& C and C \& A for SBP and DBP is highly significant $(\mathrm{p}<0.001)$.

Table 3:

\begin{tabular}{|c|c|c|c|}
\hline & Group A & Group B & Group C \\
\hline SBP $($ Mean \pm SE) & $112.63 \pm 5.909$ & $110.80 \pm 6.433$ & $149.27 \pm 4.787$ \\
\hline DBP(Mean \pm SE) & $70.73 \pm 4.843$ & $69.33 \pm 5.190$ & $98.80 \pm 4.120$ \\
\hline
\end{tabular}




\section{SPECIFIC INVESTIGATION:}

Total Cholesterol (mg/dl): Total cholesterol increased significantly during pregnancy than non pregnant state which is increase more significantly during pregnancy complicated with preeclampsia.

The $p$ value for $A \& B, B \& C, C \& A$ is $<0.001$ that is highly significant.

Table 4: Mean total cholesterol in study groups

\begin{tabular}{|c|c|c|c|}
\hline & Group A & Group B & Group C \\
\hline Mean \pm SEM & $213.63 \pm 3.831$ & $219.23 \pm 3.275$ & $235.37 \pm 4.125$ \\
\hline
\end{tabular}

High density lipoprotein (mg/dl) (HDL): It is seen that there is significant increase of HDL level in normal pregnancy compared to the non pregnant state. But this level falls significantly in preeclampsia which may be reason for the atherosclerosis like features mentioned in preeclampsia. The $\mathrm{p}$ value for $\mathrm{A} \& \mathrm{~B}, \mathrm{~B} \& \mathrm{C}, \mathrm{C} \& \mathrm{~A}$ is $<0.001$ that is highly significant.

Table 5: Mean HDL concentration among study groups

\begin{tabular}{|c|c|c|c|}
\hline & Group A & Group B & Group C \\
\hline Mean \pm SEM & $46.37 \pm 2.393$ & $60.28 \pm 3.20$ & $41.752 \pm 3.4081$ \\
\hline
\end{tabular}

Low density lipoprotein(mg/dl)(LDL): LDL is most harmful lipoprotein in our body .In table 6 it is shown that in this study LDL concentration was increased significantly during pregnancy which was further increased significantly when pregnancy was complicated with preeclampsia.

Table 6: Mean LDL concentration in the study groups

\begin{tabular}{|c|c|c|c|}
\hline & Group A & Group B & Group C \\
\hline Mean \pm SEM & $103.67 \pm 2.868$ & $114.27 \pm 3.188$ & $135.97 \pm 3.659$ \\
\hline
\end{tabular}

The $\mathrm{p}$ value for $\mathrm{A} \& \mathrm{~B}, \mathrm{~B} \& \mathrm{C}, \mathrm{C} \& \mathrm{~A}$ is $<0.001$ that is highly significant.

Very low density lipoprotein (VLDL) (mg/dl): In table 7 it is seen that VLDL was increased significantly in normal pregnancy compared non pregnant state which was significantly increased in pre-eclamptic women.

Table 7: Mean VLDL concentration among the study groups

\begin{tabular}{|c|c|c|c|}
\hline & Group A & Group B & Group C \\
\hline Mean \pm SEM & $24.63 \pm 2.107$ & $42.45 \pm 1.525$ & $53.885 \pm 1.1514$ \\
\hline
\end{tabular}

The $\mathrm{p}$ value for $A \& B, B \& C, C \& A$ is $<0.001$ that is highly significant. 


\section{ORIGINAL ARTICLE}

Triglyceride (mg/dl): On calculating the mean triglyceride concentration among the three groups it is seen that mean triglyceride level was significantly increased in pregnant females as compared non pregnant females but it was more significantly increased in pre-eclamptic mothers.

Table 8: Mean triglyceride level among the study groups

\begin{tabular}{|c|c|c|c|}
\hline & Group A & Group B & Group C \\
\hline Mean \pm SEM & $120.17 \pm 3.465$ & $215.20 \pm 1.645$ & $273.12 \pm 8.108$ \\
\hline
\end{tabular}

The $p$ value for $A \& B, B \& C, C \& A$ is $<0.001$ that is highly significant.

DISCUSSION: Endothelial dysfunction is the most important event in the pathogenesis of preeclampsia, and lipids have a role on this event. Free fatty acid flow, triglycerides, LDL, total lipid, cholesterol and VLDL values are increased during preeclampsia. Beta oxidation is disturbed in the liver and VLDL, LDL and triglycerides are stored in liver. Lipid peroxidase and cytokines increase secondary to an increase at the levels of plasma lipids. Endothelial cells are disturbed directly or indirectly and vasoconstriction occurs all throughout the body. $(8,12)$

This study was conducted over a period of one year. All the three groups in this study were comparable in terms of age, religion and socioeconomic. Two pregnant groups were carefully chosen at same gestational age. Different lines of evidence indicate that abnormal lipid metabolism is not a mere manifestation but also pathogenesis of disease. High density lipoprotein is one of the four major plasma lipoprotein classes that are involved in lipid metabolism and the exchange of cholesterol esters and triacylglycerol between tissues. HDL cholesterol facilitate reverse cholesterol transport by caring excess, potentially harmful cholesterol from peripheral tissue to liver .It also involve in activating lipoprotein which releases fatty acid that can be oxidized to provide energy. In this study pre-eclamptic pregnant mother have been found to have highly significantly $(\mathrm{p}<.0001)$ decreased level serum HDL cholesterol than normotensive pregnant mother and non-pregnant woman. A study in Bangladesh showed a significant decrease in HDL-C in pre-eclamptic and eclamptic pregnant women. Oestrogen is responsible for induction of TG and HDL and suppression of serum LDL and oestrogen level falls in preeclampsia $(6,9)$. The Low level of HDL in preeclampsia is however not only because of hypo-oestrogenaemia but also due to insulin resistance (10).In this study there is significant increase of HDL in pregnancy than non pregnant state. Potter et al also reported similar results, in their study they show, HDL levels increased during pregnancy. In a study in chronic hypertensive pregnants, the levels of HDL, LDL and total lipid were lower compared to the normotensive group, but total cholesterol and triglyceride levels were not different in the two groups. (11)

In a study in Portuguese woman, subjects with preeclampsia had a lower HDL cholesterol level compare to healthy pregnant woman. Another study in turkey shows same observation. Similarly, Rubin aziz and Tabassum Mehboob conducted a study and result shows HDL cholesterol decreased significantly in preeclampsia. Belo et al in their study concluded that pre-eclamptic women exhibited, in third trimester, higher mean serum triglyceride concentration and low HDL cholesterol.

In our study serum triglyceride level was significantly increased in preeclampsia and the result is similar to Hubel et al which showed that triglyceride and free fatty acids increased two-fold 


\section{ORIGINAL ARTICLE}

in pre-eclamptic patients but HDL, LDL and total cholesterol levels were not different from the normotensive pregnant women, whereas in our study LDL and total cholesterol levels were significantly higher in the pre-eclamptic patients. (13).

The association of LDL concentration with preeclampsia is biologically plausible. It has been that oxidized LDL increased sensitivity to the presser agents and inhibit epithelial dependent vasodilatation.

Garzetti et al found that lipid fractions increased in pre-eclamptic patients and plasma lipid peroxidase and free radicals were activated. Also platelet and erythrocyte membrane antioxidant systems were inactivated and this had an important role in etiopathogenesis. (14) In normal pregnancies lipolysis increases but in pre-eclamptic patients this increase is significantly higher. The changes of lipid metabolism and free fatty acids are not etiological factors but have important role in the pathogenesis. (15)

Increased TG, found in pregnancy induced hypertension, is likely to be deposited in predisposed vessels, such as the uterine spiral arteries and contributes to the endothelial dysfunction, both directly and indirectly through generation of small, dense LDL (16). Moreover, this hypertriglyceridemia may be associated with hypercoagulability (17)

In our study VLDL concentration was significantly increased in normal pregnancy which is further significantly increased in preeclampsia .This result is supported by some researchers, they showed that VLDLC level, might rise up to 2.5 folds at term over the pre-pregnancy level $(18,19)$. VLDL level further increase in PIH as evidenced in the present study in corroboration with those of other workers (18), perhaps due to increased VLDL lipoproteins which accumulate over the maternal vascular endothelium, particularly those of uterine and renal vessels (20). Further VLDL may cause injury to the endothelium, while a specific toxicity- preventing-activity-protein protects against the VLDL-induced injury in the pathogenic process of toxaemia (21)

In our study total cholesterol was significantly increased in preeclampsia. Similarly many studies have found significant increase in serum TC in toxaemia of pregnancy $(14,22)$. In India Usha Adiga et al. conducted study at Mangalore which shows that 20\% increased in total cholesterol, 27\% decreased in HDL cholesterol levels. They also stated that hypercholesterolemia promoted the formation of free radicals.

CONCLUSION: We conclude that all lipid fractions alter during pregnancy in both normotensive and hypertensive patients. In hypertensive pregnant women, hyperlipidemia is more profound than in the normotensive group. The findings reported in this study, suggest that the women who develop pre-eclampsia had disturbed lipid profile due to abnormal lipid metabolism .This association may be significant in understanding the pathological process of preeclampsia and may help in diagnosis of various causative factor and also developing strategies for prevention and early diagnosis of preeclampsia and eclampsia.

\section{REFERENCES:}

1. Cengiz C, Kimya Y. Maternal Fizyoloji. Temel Kadın Hastalıkları ve Dogum Bilgisi (Eds Kisnisçi et al) Günes, Ankara.1997; 242-243.

2. Chapman JF, Tsongalis GJ. Pregnancy and fetal development. In: Kaplan LA, Pesce AJ, eds. Clinical Chemistry. Missouri: Mosby; 1996:799-810. 


\section{ORIGINAL ARTICLE}

3. Herrera E, Gomez DC, Lasuncion MA. Lipid metabolism in pregnancy. Biol Neonate. 1987; 52(2):70-77.

4. Dutta, D.C. (2001) Hypertensive disorders in pregnancy, In: Textbook of Obstetrics, Ed. Konar, H.L., 5th edition, New Central Book Agency, Kolkata, p 234-55.

5. Robson, S.C. (1999) Hypertension and renal disease in pregnancy, In: Dewhurst's Textbook of Obstetrics and Gynaecology for postgraduates, Ed. Edmonds, D.K., 6 ${ }^{\text {th }}$ edition, Blackwell Science Ltd., New York, p 167- 9.

6. Gractacose E, Casals E, Gomez O, Llurba E, Mercader I, Carach V, et al. Increased susceptibility to low density lipoprotein oxidation in women with a history of pr-eclampsia. Br J Obstet Gynaecol. 2003; 110(4):400-4.

7. Caren G, Solomon, Seely EW. Preeclampsia searching for cause. New Eng J Med. 2004; 350(7):641-2.

8. Enquobahrie DA, Williams MA, Butler CL, Frderick IO, Miller RS, Luthy DA. Maternal plasma lipid concentration in early pregnancy and risk of preeclampsia. Am J Hypertens. 2004; 17(7):574-81.

9. Bradley R, Crook D. Pregnancy, oral contraception and hormone replacement therapy. In: Textbook of Clinical Biochemistry: Metabolic and Clinical aspects. Marshall WJ, Bangert SK, editors.1st edition, Churchill Livingstone, London, 1995. p. 413-22.

10. Kaaja R, Tirkkanen MJ, Viinnkka L, Ylikorkala O. Serum lipoproteins, insulin and urinary prostanoid metabolites in normal and hypertensive pregnant women. Obstet Gynecol. 1995; 85(3):353-6.

11. Potter MJ, Nestel JP. The hyperlipidemia of pregnancy in norma8. Sattar N, Gaw A, Packard JC et al. Potential pathogenic roles of aberrant lipoprotein and fatty acid metabolism in preeclampsia. Br J Obstet Gynecol 1996;103:614-620.

12. Van Den Elzen HJ, Wladimiroff J, Cohen-Overbeek TE et al. Serum lipids in early pregnancy and risk of preeclampsia. Br J Obstet Gynecol. 1996;103:117-122.l and complicated pregnancies. Am J Obstet Gynecol.1979; 15:165-170.

13. Hubel CA, McLaughi MK, Evans RV et al. Fasting serum triglycerides free fatty acids and malondialdehyde are increased in preeclampsia are positively correlated with in 48 hours postpartum. Am J Obstet Gynecol. 1996; 174(3):975-982.

14. Garzetti GG, Tranquilli AL, Cugini AM et al. Altered lipid composition increased lipid peroxidation and altered fluidity of the membrane as evidence of platelet damage in preeclampsia. Obstet Gynecol. 1993; 81:337-340.

15. Sibai MB. Immunologic aspects of preeclampsia. Clin Obstet Gynecol. 1991; 34:27-34.

16. Sattar N, Bendomir A, Berry C, Shepherd J, Greer IA, Packard CJ. Lipoprotein sub fraction concentrations in preeclampsia: pathogenic parallels to atherosclerosis. Obstet Gynecol. 1997; 89(3):403-8.

17. Kokia E, Barkai G, Reichman B, Segal P, Goldman B, Mashiach S. Maternal serum lipid profile in pregnancies complicated by hypertensive disorders. J Perinat Med. 1990; 18(6):473-8.

18. Teichmann, A.T., Wieland, H., Cremer, P., Knlow, G. And Mehle, U. (1988) Serum lipid and lipoprotein concentrations in pregnancy and at onset of labour in normal and complicated pregnancies caused by hypertensive gestosis and fetal growth retardation. Geburtshilfe Frauenheilkd (Germany, West) 48(3), 134-9. 


\section{ORIGINAL ARTICLE}

19. Knopp, R.H., Warth, M.R., Charles, D., Childs, M., Li, J.R., Mabuchi, H. and Von, M.I.A. (1986) Lipoprotein metabolism in pregnancy, fat transport to the fetus and the effects of diabetes, Biol. Neonate (Switzerland) 50(6), 297-317.

20. Potter, J.M. and Netel, P.J. (1979) The hyperlipidemia of pregnancy in normal and complicated pregnancies. Am. J. Obstet. Gynaecol. (United States) 133(2), 165-70.

21. Arbogast, B.W., Leeper, S.C., Merrick, R.D., Olive, K.E. and Taylor, R.N. (1994). Which plasma factors bring about disturbance of endothelial function in pre-eclampsia. Lancet 343(8893), 340-1.

22. Adegoke OA, Iyare EE, Gbenebitse SO. Fasting plasma glucose and cholesterol levels in pregnant Nigerian women. Niger Postgrad Med J. 2003; 10(1):32-6.

\section{AUTHORS:}

1. Dhrubajyati Saha

2. Pampa Roy

3. Rajib Pal

4. Tapan Kumar Ganguli

5. Santu Maity

6. Bibek Mohan Rakshit

7. Prabir Sengupta

8. Sanjana Halder

\section{PARTICULARS OF CONTRIBUTORS}

1. Senior Resident, Department of Obstetrics and Gynaecology, Chittaranjan Seva Sadan.

2. Senior Resident, Department of Pediatrics, Dr. B.C Roy Postgraduate Institute of Pediatric Sciences.

3. Assistant Professor, Department of Obstetrics and Gynaecology, Burdwan Medical College.

4. Senior Resident, Department of Obstetrics and Gynaecology, Burdwan Medical College.
5. Junior Resident, Department of Obstetrics and Gynaecology, Burdwan Medical College.

6. Associate Professor, Department of Obstetrics and Gynaecology, Burdwan Medical College.

7. Professor, Department of Obstetrics and Gynaecology, Burdwan Medical College.

8. Assistant Professor, Department of Obstetrics and Gynaecology, Chittaranjan Seva Sadan.

\section{NAME ADRRESS EMAIL ID OF THE CORRESPONDING AUTHOR:}

Dr. Dhrubajyati Saha, C/O Gouranga lal Saha, North Habra, Post Offish Road, P.O - Habra, North 24,

Parganas, West Bengal, PIN - 743263.

Email - dhrubajyati1984@gmail.com

Date of Submission: 28/07/2013.

Date of Peer Review: 29/07/2013.

Date of Acceptance: 01/08/2013.

Date of Publishing: 05/08/2013 